\title{
Author Index
}

Ammar, El-Desouky; Nault, L.R.: Assembly and accumulation sites of maize mosaic virus in its plant-hopper vector 33-41

Anderson, D.A.; Locarnini, S.A.; Coulepis, A.G.; Gust, I.D.: Restrictive events in the replication of hepatitis A virus in vitro 26-32

Bashiruddin, John B., see Reinganum, Carl 10-17 Bia, Frank J.; Lucia, Helen L.; Bia, Margaret Johnson; Vine, William; Tuttle, Ann: Effects of cyclo-sporine on the pathogenesis of primary cyto-megalovirus infection in the guinea pig 154-165 Bia, Margaret Johnson, see Bia, Frank J. 154-165 Bishop, David H.L.: The genetic basis for describing viruses as species 79-93 Bosman, F., see Bruggeman, C.A. 1-9 Brinton, M.A., see Westaway, E.G. 125-139, 183-192 Brown, F.: The species issue in virology 61 Bruggeman, C.A.; Meijer, H.; Bosman, F.; van Boven, C.P.A.: Biology of rat cytomegalovirus infection 1-9 Burrell, C.J., see Gowans, E.J. 220-225

Cai, Rifen, see Wang, Shusheng 140-146 Callahan, Sharon M., see Carp, Richard I. 170-173 Carballal, Guadalupe, see Dulout, Fernando N.

193-198 Carp, Richard I.; Callahan, Sharon M.: Effect of prior treatment with thioglycolate on the incubation period of intraperitoneally injected scrapie 170-173 Chen, Jie, see Wang, Shusheng 140-146 Conti, C.; Tsiang, H.: Effect of concanavalin A on the early events of rabies virus infection of CER cells 166-169 Coulepis, A.G., see Anderson, D.A. 26-32 Cross, Geoffrey F., see Reinganum, Carl 10-17

De Luca, Julio C, see Dulout, Fernando N. 193-198 Dubsky, Margaret, seeSheinin, Rose 174180 Dulout, Fernando N.; Panisse, Horacio E.; Carballal, Guadalupe; von Guradze, Huberto N.; De Luca, Julio C; Oubiña, José R.; Videla, Cristina: Junin virus-induced chromosomal aberrations in the guinea pig. Synergism between the attenuated strain XJ-clone 3 and caffeine 193-198

Espinoza, Eduardo, Farias, Gilda; Soler, Maritza; Kuznar, Juan: Identity between infectious pancreatic necrosis virus VR-299 and a Chilean isolate 58-60

Fabbro, Julie, see Sheinin, Rose 174-180

Falke, D.; Knoblich, A.; Müller, S.: Fusion from

without induced by herpes simplex virus type 1

211-219 Farias, Gilda, see Espinoza, Eduardo 58-60

Gaidamovich, S.Ya., see Westaway, E.G. 125-139, 183-192

Gentry, G.A., see Veerisetty, V. 42-49

Gowans, E.J.; Burrell, C.J.; Jilbert, A.R.; Marmion, B.P.: Cytoplasmic (but not nuclear) hepatitis $B$ virus (HBV) core antigen reflects HBV DNA synthesis at the level of the infected hepatocyte 220-225

Gust, I.D., see Anderson, D.A. 26-32

Harrison, Bryan D.: Usefulness and limitations of the species concept for plant viruses 71-78

Horzinek, M.C., see Westaway, E.G. 125-139, 183-192 
Huang, Chen-hsiang; Liang, Hsueh-chia; Jia, Feng-Ian: Beneficial role of a nonpathogenic orbilike virus: studies on the interfering effect of M14 virus in mice and mosquitoes infected with Japanese encephalitis virus 147-153

Igarashi, A., see Westaway, E.G. 125-139,183-192

Jarrett, William F.H., see Moar, Martin H. 108-118 Jia, Feng-Ian, see Huang, Chen-hsiang 147153 Jiang, Rensheng, see Wang, Shusheng 140-146 Jilbert, A.R., see Gowans, E.J. 220-225

Kääriäinen, L., see Westaway, E.G. 125-139,183-192 Kern, Earl R., seeStanberry, Lawrence R. 226-231 Kingsbury, D.W.: Species classification problems in

virus taxonomy 62-70 Kislev, Naomi: DNA homology relationships between Spodoptera

littoralis nuclear polyhedrosis virus and other baculoviruses 50-57

Author Index

233

Knoblich, A., see Falke, D. 211-219 Koivisto, Vesa, see Lehtinen, Matti 18-25 Kuznar, Juan, see Espinoza, Eduardo 58-60

Lehtinen, Matti; Koivisto, Vesa; Lehtinen, Tuula; Paavonen, Jorma; Leinikki, Pauli:

Immunoblot-ting and enzyme-linked immunosorbent assay analysis of serological responses in patients infected with herpes simplex virus types 1 and 2 18-25 Lehtinen, Tuula, see Lehtinen, Matti 18-25 Leinikki, Pauli, see Lehtinen, Matti 18-25 Li, Rongjian, see Wang, Shusheng 140146 Liang, Hsueh-chia, see Huang, Chen-hsiang 147-153 Locarnini, S.A., see Anderson, D.A. 26-32 Lucia, Helen L., see Bia, Frank J. 154-165 Lvov, D.K., see Westaway, E.G. 125-139,183192

Marmion, B.P., seeGowans, E.J. 220-225

Meijer, H., see Bruggeman, C.A. 1-9

Milne, R.G.: Alternatives to the species concept for virus taxonomy 94-98

Moar, Martin H.; Jarrett, William F.H.: A cutaneous fibropapilloma from a red deer (Cervus elaphus) associated with a papillomavirus 108-118

Müller, S., see Falke, D. 211-219

Nault, L.R., seeAmmar, El-Desouky 33-41 Nielsen, CM., see Wang, K.-Q. 99-107

Oubiña, José R., see Dulout, Fernando N. 193-198 Overall, James C., Jr., see Stanberry, Lawrence R.

226-231

Paavonen, Jorma, see Lehtinen, Matti 18-25 Panisse, Horacio E., see Dulout, Fernando N. 193198 Porterfield, J.S., see Westaway, E.G. 125-139,183-192

Reinganum, Carl; Bashiruddin, John B.; Cross, Geoffrey F.: Boolarra virus: a member of the Noda-viridae isolated from Oncopera intricoides (Lepid-optera: Hepialidae) 10-17

Richards, James T., see Stanberry, Lawrence R. 226-231

Russell, P.K., see Westaway, E.G. 125-139,183-192

Sarov, Israel, see Szanton, Esther 119-124 Sheinin, Rose; Fabbro, Julie; Dubsky, Margaret:

Mouse polyoma virus and adenovirus replication

in cells temperature-sensitive in DNA synthesis 174-180 Soler, Maritza, see Espinoza, Eduardo 58-60 Stanberry, Lawrence R.; Kern, Earl R.; Richards, James T.; Overall, James C, Jr.:

Recurrent genital herpes simplex virus infection in guinea pigs 226-231 Szanton, Esther; Sarov, Israel: Interaction between polymorphonuclear leukocytes and varicella-zoster virus-infected cells 119-124 
Trent, D.W., see Westaway, E.G. 125-139,183-192 Tsiang, J., see Conti, C. 166-169 Tuttle, Ann, see Bia, Frank J. 154-165

van Amstel, Peter J., see van den Berg, Frank M.

199-210 van Boven, C.P.A., see Bruggeman, C.A. 1-9 van den Berg, Frank M.; van Amstel, Peter J.; Wal-boomers, Jan M.M.: Construction of rat cell lines that contain potential morphologically transforming regions of the herpes simplex virus type 2 genome 199-210 Veerisetty, V.; Gentry, G.A.: HSVl-specificthymidylate kinase activity in infected cells 42-49 Vestergaard, B.F., see Wang, K.-Q. 99-107 Videla, Cristina, see Dulout, Fernando N. 193-198 Vine, William, see Bia, Frank J. 154-165 von Guradze, Huberto N., see Dulout, Fernando N. 193-198

Walboomers, Jan M.M., see van den Berg, Frank M. 199-210

Wang, K.-Q.; Nielsen, CM.; Vestergaard, B.F.: Isolation and adaptation characteristics of hepatitis A virus in primary African green monkey kidney cells: production of antigen useful for ELISA se-rology 99-107

Wang, Shusheng; Cai, Rifen; Chen, Jie; Li, Rongjian, Jiang, Rensheng: Etiologic studies of the 1983 and 1984 outbreaks of epidemic diarrhea in Guangxi 140-146

Westaway, E.G.; Brinton, M.A.; Gaidamovich, S.Ya.; Horzinek, M.C.; Igarashi, A.; Kääriäinen, L.; Lvov, D.K.; Porterfield, J.S.; Russell, P.K.; Trent, D.W.: Togaviridae 125-139

Westaway, E.G.; Brinton, M.A.; Gaidamovich, S.Ya.; Horzinek, M.C; Igarashi, A.; Kääriäinen, L.; Lvov, D.K.; Porterfield, J.S.; Russell, P.K.; Trent, D.W.: Flavivirídae 183-192 